\title{
REVIEW
}

\section{The UK Hib vaccine experience}

P T Heath, J McVernon

Haemophilus influenzae type $b$ (Hib) is an important pathogen in children $<5$ years of age. The Hib conjugate vaccines were the first polysaccharideprotein conjugate vaccines to be used in routine childhood schedules. Their introduction in the UK in 1992 has resulted in the near elimination of $\mathrm{Hib}$ disease. The UK Hib vaccine programme has a number of unique features including an accelerated schedule, absence of a booster dose, and the inclusion of a catch up component at the beginning of the programme. Collaboration between UK paediatricians, microbiologists, and public health physicians has allowed active national surveillance of Haemophilus influenzae disease and enabled important conclusions to be drawn. These include high vaccine effectiveness, the presence of herd immunity, and the frequency of risk factors in cases of vaccine failure and in cases of invasive disease due to non-b $H$ influenzae. Parallel studies have shown the immunogenicity, induction of immunological memory, and persistence of immunity following vaccination at the UK schedule, as well as measuring the impact of vaccination on pharyngeal Hib carriage. Cases continue to occur and complacency is ill advised, particularly as other vaccines and combinations are introduced. Surveillance of $H$ influenzae disease in the UK remains important.

See end of article for authors' affiliations

Correspondence to: Dr P T Heath, Department of Child Health and St George's Vaccine Institute, St George's Hospital Medical School, Cranmer Terrace, London SW 17 ORE, UK

pheath@sghms.ac.uk

Accepted 4 March 2002
D octors training in paediatrics in the UK in the year 2002 are unlikely to see a case of epiglottitis. Nor are they likely to see cases of meningitis, cellulitis, osteomyelitis, or septic arthritis caused by the bacterium Haemophilus influenzae type b (Hib). Yet prior to $1992 \mathrm{Hib}$ was the most common cause of meningitis in childhood, almost the exclusive cause of epiglottitis, and a common cause of cellulitis and bone and joint infections. The introduction of Hib conjugate vaccines in the UK has almost eliminated this pathogen. What lessons has the UK Hib vaccine experience taught us and what issues remain?

\section{THE BACTERIUM AND THE VACCINE}

Haemophilus influenzae (Hi) was first identified as a pathogen in 1883 by Robert Koch, who described small Gram negative rods in pus from patients with conjunctivitis. ${ }^{1}$ In 1931 Margaret Pittman $^{2}$ identified six antigenically distinct encapsulated strains defined as serotypes a to $\mathrm{f}$, as well as non-encapsulated (non-typeable) strains.
She recognised that one capsular type, serotype $b$, was responsible for almost all cases of meningitis caused by Hi. Subsequent studies confirmed that Hi type b strain (Hib) was responsible for approximately $95 \%$ of all invasive $\mathrm{Hi}$ disease in children. ${ }^{34}$

Epidemiological work in the 1980s and 1990s evaluated the burden of Hib disease in UK children. Studies performed in different parts of the UK indicated incidence rates ranging from 21 to 44 per $100000<5$ years of age, ${ }^{35-7}$ a peak age of 6-7 months, ${ }^{3}$ and case fatality rates of 2.44.3\%. ${ }^{35}$ Extrapolation from these data suggested that up to 1500 cases of invasive Hib disease occurred in the UK every year, of which 900 were cases of meningitis and 60 resulted in death. ${ }^{3}$ Sequelae of Hib meningitis include mental retardation in $6 \%$, seizures in $6 \%$, and profound or severe deafness in $7 \%$ of survivors. ${ }^{8}$ This significant burden of disease was a major impetus for vaccine introduction.

The first vaccine to be developed against Hib was composed of the organism's polysaccharide capsule, polyribosylribitol phosphate (PRP). An early trial of this vaccine showed $>90 \%$ efficacy in children who received vaccine aged 18 months or older, but no protection in younger children. ${ }^{9}$ The explanation lies in the poor immunogenicity of PRP in the infant and its failure to induce immunological memory, both features of $\mathrm{T}$ cell independent antigens such as polysaccharides. The chemical attachment (conjugation) of PRP to protein antigens, such as diphtheria and tetanus toxoids, was the basis of the development of Hib conjugate vaccines. Conjugates enlist the help of $\mathrm{T}$ cells through their protein component, allowing long term recall immunity as well as inducing antibody production in young infants. The efficacy of Hib conjugate vaccines was shown in trials performed in Finland and the USA (summarised in Heath ${ }^{10}$ ).

In 1991 a controlled intervention study of one conjugate, PRP-T (PRP conjugated to tetanus toxoid) began in the Oxford health region. ${ }^{11}$ PRP-T was given to infants in four of the region's eight districts and not offered to infants in the other four districts until October 1992. None of the infants given three doses developed Hib disease, whereas 11 cases were observed in the control population, a vaccine efficacy of $100 \%$ (95\% CI $80-100 \%) .{ }^{12}$ This was the first controlled intervention study of this vaccine to be completed and provided confidence that its use in the UK national immunisation programme would be successful.

Abbreviations: BPSU, British Paediatric Surveillance Unit; $\mathrm{Hi}$, Haemophilus influenzae; Hib, Haemophilus influenzae type b; PRP, polyribosylribitol phosphate; PRP-T, PRP conjugated to tetanus toxoid 
Table 1 Incidence of Hib disease in UK children $<5$ years of age, January 1996 to December 2000

\begin{tabular}{lll}
\hline Year & Cases & Incidence rate per $10^{5}(95 \% \mathrm{Cl})$ \\
\hline 1996 & 31 & $0.83(0.53,1.1)$ \\
1997 & 31 & $0.84(0.54,1.1)$ \\
1998 & 23 & $0.63(0.37,0.89)$ \\
1999 & 39 & $1.1(0.74,1.4)$ \\
2000 & 66 & $1.8(1.4,2.3)$ \\
\hline
\end{tabular}

\section{VACCINE IMPLEMENTATION IN THE UK}

Routine immunisation with Hib conjugate vaccines was introduced in the UK in October 1992 according to the primary schedule of three doses at 2,3, and 4 months of age. Compared with Hib programmes elsewhere, there were three unique features about the UK approach: primary vaccination was given earlier with completion by 4 months of age; a fourth (booster) dose of Hib vaccine was not given; and vaccination was offered to all children up to 48 months of age in a nationwide "catch up" programme planned to occur over the first year of implementation. The rationale for the UK approach was the belief that immunological memory after three doses in infancy would be sufficient for protection through the early childhood years when susceptibility was greatest. Administration of a single dose to children was proposed to reduce carriage and thereby transmission of Hib in the childhood population. In order to monitor the impact of this distinct programme, a surveillance study was initiated under the auspices of the British Paediatric Surveillance Unit (BPSU) in collaboration with the Haemophilus Reference Unit of the Public Health Laboratory Service.

Hib vaccine coverage has been relatively high since its introduction in 1992. COVER data indicate that between 1993 and 2000 an average of $92.5 \%$ of infants received three doses by 12 months of age (COVER data, published quarterly in the Communicable Disease Report CDR Weekly). Details on uptake of the "catch up" programme are more limited; one study in the North East Thames region indicated that $89 \%$ and $87 \%$ of children born in 1992 and 1991 respectively had received a catch up dose as planned. ${ }^{13}$

The incidence of invasive Hib infections fell dramatically with the implementation of the vaccine programme and has remained at very low rates. Table 1 shows the incidence of Hib disease in the UK in children $<5$ years of age for the years 1996-2000. These figures contrast with the rate of 31-36/ 100000 prevaccination $^{37}$; a 98\% reduction by 1998 .

Using historical data on rates of Hib disease, it is possible to calculate age specific rates of Hib vaccine effectiveness ${ }^{14}$ (see table 2).

\section{PERSISTENCE OF PROTECTION AGAINST Hib DISEASE}

Most other countries introducing Hib vaccine into routine vaccination schedules have done so with the addition of a booster dose in the second year of life. The major reason for this is the observation that vaccine induced antibody wanes over time, ${ }^{15}$ leading to the concern that this may be accompanied by an increase in susceptibility to disease. The relation between antibody to PRP and clinical protection is inferred from studies of natural immunity, passive immunisation, and the plain unconjugated PRP vaccine. From such studies arose the serum concentrations of $0.15 \mu \mathrm{g} / \mathrm{ml}$ as a correlate of short term protection, and $1.0 \mathrm{\mu g} / \mathrm{ml}$ as a correlate of long term protection against disease. ${ }^{16}$ The relevance of these concentrations for children who have been primed for memory responses is less certain. ${ }^{17}$ In the presence of immunological memory, a rapid and protective increase in antibody concentration may be achieved in response to exposure to the organism, despite a low background antibody concentration.

A study was performed to document the persistence of vaccine induced antibody in UK children. This confirmed the decline over the first five years of life. By 72 months of age, $32 \%$ of children (95\% CI 21 to $46 \%$ ) had concentrations $<0.15$ $\mathrm{\mu g} / \mathrm{ml}$ and therefore were apparently unprotected..$^{14}$ In the absence of surveillance data, one might conclude that a second year booster dose was imperative in order to prevent this apparent decline in protection. However, the BPSU study afforded the unique opportunity to interpret this in the light of clinical vaccine failure cases. ${ }^{14}{ }^{18}$ Absolute estimates of vaccine effectiveness up to 6 years of age remain high, with only a small decline in protection over the first five years of life (table 2).

Immunological memory has been shown in children receiving the UK schedule ${ }^{19}$; this may explain the apparent disparity between high clinical protection despite low measured antibody concentrations. An additional explanation is "herd immunity" resulting from the impact of widespread vaccination on reducing Hib carriage and transmission.

\section{Hib CARRIAGE IN THE UK}

The nasopharynx is the natural niche for Hib, and in the prevaccine era the peak age of carriage was in young children. Hib conjugate vaccines delay or prevent acquisition of carriage of the organism. ${ }^{20}{ }^{21}$ Transmission is thereby reduced and a herd immune effect is produced, leading to a greater reduction in disease incidence than would be expected from immunisation alone. ${ }^{22}{ }^{23}$ In Oxfordshire between 1991 and 1994 a reduction in carriage from $6.7 \%$ to $1.3 \%$ was observed in 4 year old children. ${ }^{24}$ Studies in nursery school children in 1991, 1994, and 1996 also indicated substantial declines in carriage $(4.0 \%$, $0.7 \%$, and $<1.0 \%$ respectively, Mary Ramsay, personal communication). Serum antibody concentrations have been shown to have a dose dependent effect on the density of colonisation. ${ }^{21}$ Transudation of IgG across mucosal surfaces has been implicated in this process as have mucosally produced antibodies. ${ }^{25}$ A recent study in the Dominican Republic showed a direct relation between serum anticapsular antibody concentrations achieved after vaccination and carriage. Those children achieving concentrations $>5.0 \mu \mathrm{g} / \mathrm{ml}$ were significantly less likely to be carriers at 9 months of age. ${ }^{26}$ The impact of herd immunity can be seen by the tenfold reduction in Hib disease rates in unvaccinated children $<1$ year of age in 1998 compared with rates in similarly aged children before vaccination began. ${ }^{14}$ Another reflection of herd immunity is the impact of childhood Hib vaccination on adult Hib disease. A review of adult cases of Hib disease in five English regions between 1990 and 1995 showed a halving of case numbers between the first three year period and the last two year period. ${ }^{27}$

Thus it is apparent that two mechanisms are operating in UK vaccinated children, which may explain the disparity between low antibody concentrations and high vaccine efficacy: immunological memory and herd immunity. Defining the relative importance of these two mechanisms is critical to our continued understanding of the epidemiology of Hib disease.

\section{CHARACTERISATION OF UK Hib VACCINE FAILURES}

A total of 273 confirmed reports of invasive Hib disease in vaccinated children were collected over nine years from October 1992 to the end of September 2001. Table 3 details the modes of presentation and associated medical and immunological conditions among cases. The clinical presentation of vaccine failure cases is little different to that of Hib cases in the prevaccine era, with a predominance of meningitis and epiglottitis. Mortality has decreased from the previously 
Table 2 Age specific point estimates of vaccine effectiveness

\begin{tabular}{|c|c|c|c|c|c|}
\hline Age (mth) & Exposure time (child years) & $\begin{array}{l}\text { Observed cases } \\
\text { (number of true } \\
\text { vaccine failures) }\end{array}$ & $\begin{array}{l}\text { Expected cases* } \\
\text { (number) }\end{array}$ & $\%$ effectiveness & $95 \% \mathrm{Cl}$ \\
\hline $5-11$ & 3155698 & 22 & 3437 & 99.4 & $99.0,99.6$ \\
\hline $12-23$ & 4751048 & 51 & 2376 & 97.9 & $97.2,98.4$ \\
\hline $24-35$ & 4096310 & 47 & 1037 & 95.5 & $93.9,96.7$ \\
\hline $36-47$ & 3435236 & 30 & 618 & 95.1 & $93.0,96.8$ \\
\hline $48-59$ & 2766590 & 15 & 149 & 89.9 & $82.9,94.5$ \\
\hline 60-71 & 2087099 & 3 & 102 & 97.1 & $91.2,99.4$ \\
\hline All ages & 21698320 & 171 & 6509 & 97.4 & $96.9,97.8$ \\
\hline
\end{tabular}

Table 3 Presenting illness and associated conditions of Hib vaccine failures, October 1992 to September 2001

\begin{tabular}{lclcc}
\hline $\begin{array}{l}\text { Presenting illness } \\
(n=273)\end{array}$ & Number & Associated condition & $\begin{array}{l}\text { Number of } \\
\text { evaluable cases }\end{array}$ & $\begin{array}{l}\text { Number with } \\
\text { risk factor }\end{array}$ \\
\hline Meningitis & 131 & Prematurity & 238 & 24 \\
Epiglottitis & 60 & Immunoglobulin deficiency & 143 & 39 \\
Bacteraemia & 44 & Clinical risk factor & 242 & 29 \\
Pneumonia & 14 & Malignancy & 9 \\
Cellulitis & 14 & Dysmorphic syndrome & 6 \\
Septic arthritis & 6 & Down's syndrome & 3 \\
Other & 4 & Chromosomal anomaly & 3 \\
& & Neutropenia & 1 \\
\end{tabular}

observed $4.3 \%^{3}$ in unimmunised children to $2.6 \%$ among vaccine failures. Associated medical conditions, which may confer additional susceptibility to infection, were uncommon among Hib cases in the prevaccine era. ${ }^{28}$ As Table 3 indicates, however, they are more common among vaccine failure cases. This is not surprising given the strong immunogenicity and effectiveness of the vaccine; this highlights the need to investigate children for possible predisposing causes of vaccine failure (especially antibody deficiency). What is perhaps unexpected is the fact that the majority of the children appear otherwise healthy. Subtle and specific immune deficiencies in such children may account for this. Problems with vaccine or the vaccination process are conceivable but not proven.

Of the clinical conditions listed, a history of premature delivery is the most frequent. It has been shown that vaccinated premature infants may have lower concentrations of antibody than term infants. ${ }^{29}$ Thus, an increased risk of clinical vaccine failure is possible. Data from the BPSU study indicate a trend towards increased risk of vaccine failure among premature infants (RR 1.5 versus term infants), but this does not reach statistical significance, possibly because of the small number of cases involved.

\section{NON-b Hi DISEASE IN UK CHILDREN}

Non-b Hi are a rare cause of disease in children, but in the Hib vaccine era non-b Hi have become relatively more common than type $b$ as a cause of invasive Hi disease in fully vaccinated children. ${ }^{30}$ It is important to stress that there has been no rise in absolute numbers of non-b cases in children following the introduction of vaccine. The majority of such strains are nonencapsulated (non-typeable). Compared with cases of Hib vaccine failure, vaccinated children with non-type $b$ disease tend to be younger and are less likely to present with meningitis and epiglottitis and more likely to present with pneumonia and bacteraemia. ${ }^{30}$ However, in the BPSU study, 58 (25\%) of a total of 208 cases of non-b Hi presented with meningitis, and the mortality of non-b cases was approximately $9 \%$, twice the mortality of type $b$ disease in the prevaccine era. ${ }^{3}$ The importance of verifying the strain of Hi causing disease in children is clear. Disease caused by non-b Hi may also signal an underlying host abnormality, and underlying conditions should again be sought. ${ }^{30}$ Underlying chronic disease is likely to be clinically apparent, but assessment for antibody deficiency should be considered.

\section{Hib DISEASE IN THE UK: ISSUES REMAINING}

Hib disease has increased in UK children over the past two years, with the greatest rise occurring in vaccinated children. Vaccinated children of all ages up to 5 years have been equally affected. It is possible that these fluctuations in disease incidence reflect the normal variability seen with infectious diseases. Conversely it may indicate that the Hib vaccination schedule requires revision, particularly if the desired endpoint is the elimination of Hib from the UK. Hib is a disease that may be theoretically eradicated. Man is the only host, the disease presentation is clear, and there is an effective vaccine.

Increasing disease incidence suggests continued Hib circulation. New studies of Hib carriage in susceptible age groups are required to evaluate this. Recent experience in the Alaskan native population underscores the importance of reducing Hib transmission through vaccination. A resurgence of invasive Hib cases in Alaskan native children in 1996 and 1997 was attributed to continuing Hib carriage unmasked by a change in the vaccination regime, which resulted in lower Hib antibody concentration. ${ }^{31}$

The relation between carriage and disease control is a complex one. The reduction of Hib carriage by vaccination results in greater than anticipated reductions of invasive Hib disease. However, it also removes a source of natural boosting of serum antibody concentrations, shown to occur following Hib carriage in primed individuals. ${ }^{24}{ }^{32}$ Current antibody concentrations in UK children may be predicted to be lower than in the early years of the campaign. Further seroepidemiological studies are needed to define this.

Other reasons for continued Hib circulation include under vaccination and immigration. Analysis of Hib disease in 
unvaccinated children in the BPSU study indicated that 33\% of cases were old enough to have received a complete course of vaccination and were thus preventable..$^{14}$ In addition there were a number of unvaccinated children who had recently arrived from countries in which Hib vaccine is not given. The public health message is clear: timely and complete vaccination should be attained in all children living in the UK. We must continue to strive for higher rates of completion of primary immunisation; $95 \%$ coverage should be achievable in the UK. Globally, a mere $8 \%$ of Hib disease is currently being prevented by vaccination ${ }^{33}$; it is only the industrialised countries that can afford the expensive Hib conjugate vaccines. Eradication will only be achieved by widespread vaccination in the developing world.

The need for a booster dose in the second year of life also requires constant re-evaluation. This strategy assumes that a further dose would prevent a significant proportion of cases of vaccine failure. This is by no means certain given the association of vaccine failures with other medical conditions (described earlier). However, it might raise antibody concentrations in the childhood population and thereby reduce carriage rates and Hib circulation. Further data are required, including an analysis of the cost effectiveness of such an approach. Conversely, any intervention which may lower antibody concentrations in the childhood population needs careful consideration. The lower immunogenicity of Hib vaccines when combined with acellular pertussis vaccines is a particular example and its clinical relevance has been much debated. ${ }^{34}$

The UK Hib experience has shown the extraordinary capacity of conjugate vaccines in preventing disease in children. It has also warned us that complacency is ill advised and reminded us of the basic public health principles of achieving high vaccine uptake and pursuing good disease surveillance. These principles apply equally to the introduction of the new conjugate vaccines.

Clinicians are encouraged to continue reporting cases of invasive $H$ influenzae disease to Mary Slack at the PHLS Haemophilus Reference Unit (tel: 01865 220859/220852; fax: 01865 220890; email: mary.slack@pathology-bacteriology. oxford.ac.uk), Jodie McVernon of the Oxford Vaccine Group at the John Radcliffe Hospital, Oxford (tel: 01865 221068; fax: 01865 221068; email: jodie.mcvernon@paediatrics.ox.ac.uk), or Mary Ramsay at the PHLS Communicable Disease Surveillance Centre (tel: 02082006868 ext 4085; fax: 0208200 7868; email: MRamsay@phls.org.uk).

\section{ACKNOWLEDGEMENT}

We thank Professor Robert Booy and Dr Mary Slack for helpful comments on the manuscript.

\section{Authors' affiliations}

P T Heath, Department of Child Health and St George's Vaccine Institute, St George's Hospital Medical School, London, UK J McVernon, Oxford Vaccine Group, John Radcliffe Hospital, Oxford, UK

\section{REFERENCES}

1 Koch R. Bericht uber die Thatigkeit der deutschen Cholera-kommission in Aegpytten and Ostindien. Wien Med Wochenschr 1883;33:1548-51.

2 Pittman M. Variation and type specificity in bacterial species of Haemophilus influenzae. J Exp Med 1931;53:471-92.

3 Booy R, Hodgson SA, Slack MP, et al. Invasive Haemophilus influenzae type $b$ disease in the Oxford region (1985-91). Arch Dis Child 1993;69:225-8.

4 Wenger JD, Pierce R, Deaver K, et al. Invasive Haemophilus influenzae disease: a population-based evaluation of the role of capsular polysaccharide serotype. Haemophilus Influenzae Study Group. J Infect Dis 1992;165(suppl 1):S34-5
5 Anderson EC, Begg NT, Crawshaw SC, et al. Epidemiology of invasive Haemophilus influenzae infections in England and Wales in the pre-vaccination era (1990-2). Epidemiol Infect 1995;1 15:89-100.

6 Howard AJ, Dunkin KT, Musser JM, et al. Epidemiology of Haemophilus influenzae type $b$ invasive disease in Wales. BM 1991;303:441-5.

7 Hargreaves RM, Slack MP, Howard AJ, et al. Changing patterns of invasive Haemophilus influenzae disease in England and Wales after introduction of the Hib vaccination programme. BM 1996;312:160-1.

8 Baraff LJ, Lee SI, Schriger DL. Outcomes of bacterial meningitis in children: a meta-analysis. Pediatr Infect Dis J 1993;12:389-94.

9 Peltola $\mathbf{H}$, Kayhty $\mathrm{H}$, Sivonen A, et al. Haemophilus influenzae type b capsular polysaccharide vaccine in children: a double-blind field study of 100,000 vaccinees 3 months to 5 years of age in Finland. Pediatrics 1977;60:730-7

10 Heath PT. Haemophilus influenzae type b conjugate vaccines: a review of efficacy data. Pediatr Infect Dis J 1998;17(9 suppl):S1 17-22.

11 Booy R, Taylor SA, Dobson SR, et al. Immunogenicity and safety of PRP-T conjugate vaccine given according to the British accelerated immunisation schedule. Arch Dis Child 1992;67:475-8.

12 Booy R, Hodgson S, Carpenter L, et al. Efficacy of Haemophilus influenzae type b conjugate vaccine PRP-T. Lancet 1994;344:362-6.

13 O'Brien H. Hib immunisation catch up programme in North East Thames. Commun Dis Rep CDR Rev 1994;4:R17-R18.

14 Heath PT, Booy R, Azzopardi HJ, et al. Antibody concentration and clinical protection after Hib conjugate vaccination in the United Kingdom. JAMA 2000;284:2334-40

15 Claesson BA, Schneerson R, Lagergard T, et al. Persistence of serum antibodies elicited by Haemophilus influenzae type b-tetanus toxoid conjugate vaccine in infants vaccinated at 3,5 and 12 months of age Pediatr Infect Dis J 1991;10:560-4

16 Kayhty H, Peltola H, Karanko V, et al. The protective level of serum antibodies to the capsular polysaccharide of Haemophilus influenzae type b. J Infect Dis 1983;147:1100.

17 Weinberg GA, Einhorn MS, Lenoir AA, et al. Immunologic priming to capsular polysaccharide in infants immunized with Haemophilus influenzae type b polysaccharide-Neisseria meningitidis outer membrane protein conjugate vaccine. J Pediatr 1987;111:22-7.

18 Booy R, Heath PT, Slack MP, et al. Vaccine failures after primary immunisation with Haemophilus influenzae type-b conjugate vaccine without booster. Lancet 1997;349:1 197-202

19 Goldblatt D, Miller E, McCloskey N, et al. Immunological response to conjugate vaccines in infants: follow up study. BM 1998;316:1570-1.

20 Takala AK, Eskola J, Leinonen $M$, et al. Reduction of oropharyngeal carriage of Haemophilus influenzae type $b(\mathrm{Hib})$ in children immunized with an Hib conjugate vaccine. J Infect Dis 1991;164:982-6.

21 Barbour ML, Mayon-White RT, Coles C, et al. The impact of conjugate vaccine on carriage of Haemophilus influenzae type b. J Infect Dis 1995;171:93-8.

22 van Alphen L, Spanjaard L, van der Ende A, et al. Effect of nationwide vaccination of 3-month-old infants in the Netherlands with conjugate Haemophilus influenzae type b vaccine: high efficacy and lack of herd immunity. J Pediatr 1997;131:869-73.

23 Rushdy A, Ramsay M, Heath PT, et al. Infant Hib vaccination and herd immunity. J Pediatr 1999;134:253-4.

24 Heath PT, Bowen-Morris J, Griffiths D, et al. Antibody persistence and Haemophilus influenzae type $b$ carriage after infant immunisation with PRP-T. Arch Dis Child 1997;77:488-92.

25 Kauppi M, Eskola J, Kayhty H. Anti-capsular polysaccharide antibody concentrations in saliva after immunization with Haemophilus influenzae type b conjugate vaccines. Pediatr Infect Dis J 1995;14:286-94.

26 Fernandez J, Levine OS, Sanchez J, et al. Prevention of Haemophilus influenzae type $b$ colonization by vaccination: correlation with serum anti-capsular lgG concentration. J Infect Dis 2000;182:1553-6.

27 Sarangi J, Cartwright K, Stuart J, et al. Invasive Haemophilus influenzae disease in adults. Epidemiol Infect 2000;124:441-7.

28 Tudor-Williams G, Frankland J, Isaacs D, et al. Haemophilus influenzae type $b$ disease in the Oxford region. Arch Dis Child 1989;64:517-19.

29 Washburn LK, O'Shea TM, Gillis DC, et al. Response to Haemophilus influenzae type $\mathrm{b}$ conjugate vaccine in chronically ill premature infants. $J$ Pediatr 1993;123:791-4.

30 Heath PT, Booy R, Azzopardi HJ, et al. Non-type b Haemophilus influenzae disease: clinical and epidemiologic characteristics in the Haemophilus influenzae type $b$ vaccine era. Pediatr Infect Dis J 2001;20:300-5

31 Galil K, Singleton R, Levine O, et al. Reemergence of Invasive Haemophilus influenzae type $b$ disease in a well-vaccinated population in remote Alaska. J Infect Dis 1999;179:101-6.

32 Barbour ML, Booy R, Crook DW, et al. Haemophilus influenzae type b carriage and immunity four years after receiving the Haemophilus influenzae oligosaccharide-CRM197 (HbOC) conjugate vaccine. Pediatr Infect Dis J 1993;12:478-84.

33 Peltola H. Worldwide Haemophilus influenzae type b disease at the beginning of the 21 st century: global analysis of the disease burden 25 years after the use of the polysaccharide vaccine and a decade after the advent of conjugates. Clin Microbiol Rev 2000;13:302-17.

34 Eskola J, Ward J, Dagan R, et al. Combined vaccination of Haemophilus influenzae type $b$ conjugate and diphtheria-tetanus-pertussis containing acellular pertussis. Lancet 1999;354:2063-8. 